\title{
What was the Initial Mass of Merging Black Holes in GW150914?
}

\author{
Hiromichi Tagawa ${ }^{1}$ and Masayuki Umemura ${ }^{2}$ \\ ${ }^{1}$ University of Tokyo, \\ 7-3-1 Hongo Bunkyo, Tokyo 113-0033, Japan \\ email: tagawahr@nao.ac.jp \\ ${ }^{2}$ Center for Computational Sciences, University of Tsukuba, \\ Tsukuba, Ibaraki 305-8577, Japan \\ email: umemura@ccs.tsukuba.ac.jp
}

\begin{abstract}
Recently, the Laser Interferometer Gravitational-Wave Observatory (LIGO) has detected the gravitational wave $(\mathrm{GW})$ event, GW150914, as a result of the merger of a $\sim 30 M_{\odot}$ black hole (BH) binary. So far, the merger of stellar-mass BHs has been thought to result from the evolution of binary stars. Here, we propose a novel path of the merger stemming from non-binary isolated stars. In our previous studies, we have found that multiple non-binary stellar-mass BHs whose separations are larger than 1000 AU can merge with each other under the gas-rich environments through the gas dynamical friction and three-body interaction. In this case, a considerable amount of gas can accrete onto BHs before the merger, that is, the initial mass of BHs can be lower than $30 M_{\odot}$. To explore this possibility, we perform post-Newtonian $N$-body simulations on mergers of accreting stellar-mass BHs. Based on our simulations, we find that the BH merger in GW150914 from smaller seed BHs is likely to occur in galactic nuclear regions or dense interstellar cloud cores. Furthermore, we roughly estimate event rates to be $\sim 0.4 \mathrm{yr}^{-1}$ in galactic nuclear regions and $\sim 8 \mathrm{yr}^{-1}$ in dense interstellar cloud cores.
\end{abstract}

Keywords. stars: black holes - gravitational waves - methods: numerical

\section{Introduction}

Recently, the advanced LIGO (aLIGO) observatory reported the first direct detection of GWs (Abbott et al. 2016), GW 150914. The observed signal indicates that the masses of merged BHs are $m_{1}=36_{-4}^{+5} M_{\odot}$ and $m_{2}=29_{-4}^{+4} M_{\odot}$. The merger of stellar-mass BHs has been hitherto thought to originate from inherent binary stars. However, it is unclear how such a heavy close BH binary as implied in the GW150914 event can be formed. In this paper, we newly propose a merger scenario from non-binary isolated BHs.

The multiple non-binary stellar-mass $\mathrm{BHs}$ are expected to form in first generation objects. Furthermore, according to the hierarchical merger history of galaxies, multiple non-binary stellar-mass BHs are likely to be incorporated after galaxy mergers as depicted in Figure 1. From our previous study (Tagawa et al. 2015), the multiple non-binary stellar-mass BHs whose typical extension is larger than a thousand AU can merge with each other under the gas-rich environments through the gas dynamical friction and the three-body interaction. In galaxies, we can expect several gas rich environments (Figure $1)$ such as galactic center regions of $\lesssim 1 \mathrm{pc}\left(n_{\text {gas }} \gtrsim 10^{7} \mathrm{~cm}^{-3}\right)$, dense interstellar cloud cores $\left(n_{\text {gas }}=10^{5-7} \mathrm{~cm}^{-3}\right)$, and interstellar clouds $\left(n_{\text {gas }}<10^{5} \mathrm{~cm}^{-3}\right)$. In these situations, a considerable amount of gas can accrete onto BHs before the merger (Tagawa et al. 2016). Hence, the initial mass of BHs can be lower than $30 M_{\odot}$, and the initial separation of BHs can be much larger than several AU. Therefore, mergers of non-binary isolated BHs in gas rich environments may explain the GW150914 event. In our study, to investigate 


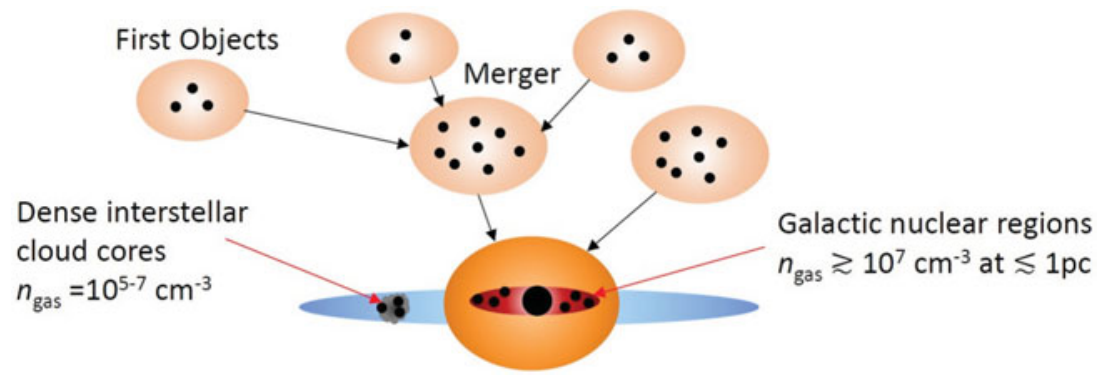

Figure 1. The formation processes of non-binary stellar-mass BHs under gas rich environments in a galaxy.

the conditions and environments under which BHs can merge from smaller mass BHs and large separation, we perform post-Newtonian N-body simulations on mergers of accreting stellar-mass BHs.

\section{Post-Newtonian N-body simulations}

Tagawa et al. (2016) gives a detailed description of the simulations used here. The equations of motion are integrated using the fourth-order Hermite scheme. The simulations incorporate the effects of gas dynamical friction, gas accretion, and the general relativity that is given by the post-Newtonian prescription up to $2.5 \mathrm{PN}$ term. $1 \mathrm{PN}$ and $2 \mathrm{PN}$ terms correspond to the pericentre shift, and $2.5 \mathrm{PN}$ term to the $\mathrm{GW}$ emission.

The key parameters in our simulations are the initial typical extension of $\mathrm{BH}$ distribution $\left(r_{\text {typ }}\right)$, the gas number density $\left(n_{\text {gas }}\right)$, and the initial BH mass $\left(m_{0}\right)$. To parameterize the accretion rate, we set the gas accretion rate $\left(\dot{m}_{i}\right)$ to be the accretion efficiency $(\epsilon \leqslant 1)$ times the Hoyle-Lyttleton accretion rate $\left(\dot{m}_{\mathrm{HL}}\right)$. The effect of the radiation pressure on Hoyle-Lyttleton accretion is incorporated as Tagawa et al. (2016).

We set the finite radius of a gas sphere whose mass is $10^{5} M_{\odot}$ and temperature is $1000 \mathrm{~K}$. We assume that two BHs merge, when their separation is less than 100 times the sum of their Schwarzschild radii. The evolution is pursued for $10 \mathrm{Gyr}$, since we consider the merger within the cosmic time. Also, we terminate the simulation, when the first $\mathrm{BH}$ merger occurs. We give the initial positions of BHs randomly in the $x-y$ plane within $r_{\text {typ }}$. Also, the velocity of each $\mathrm{BH}$ is given as the sum of a circular component and a random component. The circular velocity is given to balance against the gravity of gas in the $x-y$ plane. Besides, we give the random velocity in the $x y z$ space according to a Gaussian distribution with the same dispersion as the circular velocity.

\section{Confrontation to GW150914}

We show the results of simulations for the mergers of non-binary BHs in Figure 2. Left panel of Figure 2 shows the masses of binary BHs just before the merger in our simulations. We find several parameters in which $\mathrm{BHs}$ merge in the mass range inferred from GW150914. For these matched parameters in hatched regions, the initial BH mass is higher than $20 M_{\odot}$. Right panel of Figure 2 shows the accreted mass of binary BHs. Mostly, the accreted mass is smaller than a half of the initial $\mathrm{BH}$ mass in this range. Therefore, if the initial $\mathrm{BH}$ mass is lower than $20 M_{\odot}$, then the merger rate at the masses of GW150914 is reduced. Also, the accretion rate is expected to be around 0.01 $\dot{m}_{\mathrm{HL}}$, and the initial extension of $\mathrm{BH}$ distributions $\left(r_{\mathrm{typ}}\right)$ is to be mostly less than $1 \mathrm{pc}$. 

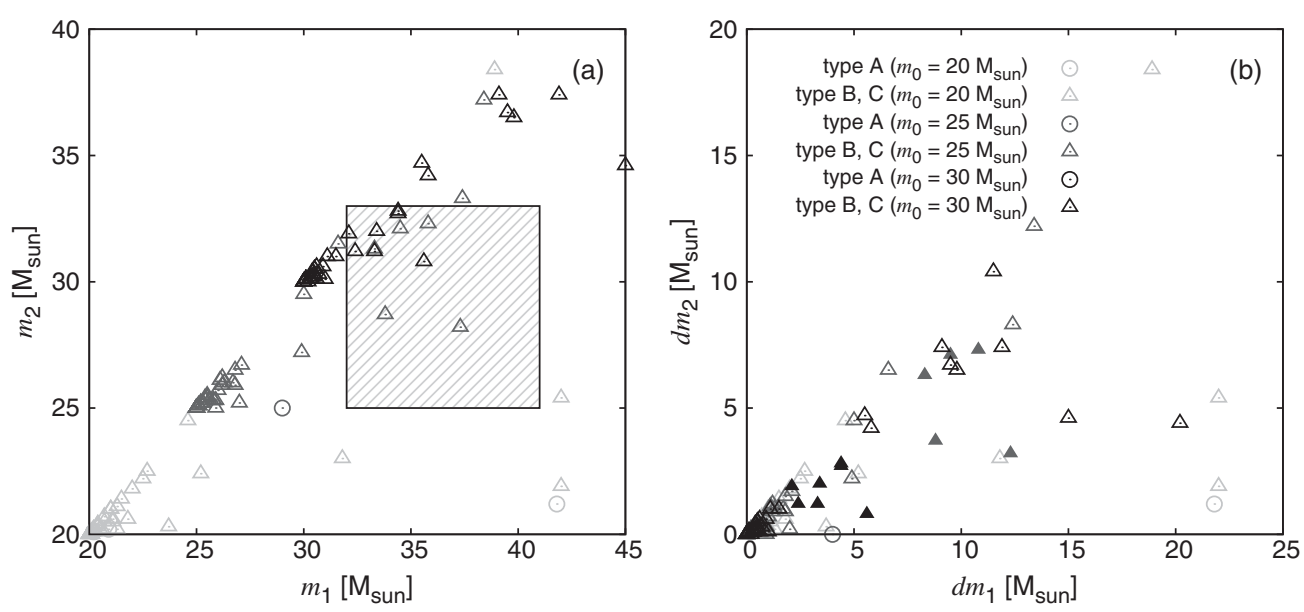

Figure 2. (a) The BH masses $\left(m_{1}>m_{2}\right)$ and (b) accreted masses in a binary just before the first merger in each run. Light gray, deep gray, black plots represent the initial masses $\left(m_{0}\right)$ of 20, 25 and $30 M_{\odot}$, respectively. Circles and triangles represent the gas drag-driven mergers (type A), and interplay-driven mergers (type B) or three body-driven mergers (type C), respectively. The masses of GW150914 with their uncertainties are indicated in panel (a) by the hatched region. The filled symbols in panel (b) are the parameters where the masses of merged BHs match those of GW150914.

In Tagawa et al. (2016), the merger mechanisms from non-binary BHs are categorized into four types: a gas drag-driven merger (type A), an interplay-driven merger (type B), a three body-driven merger (type C), and an accretion-driven merger (type D). In type A, the dynamical friction by gas effectively decays the orbit before the GW radiation drives the merger. In both types $\mathrm{B}$ and $\mathrm{C}$, the strong disturbance of the orbit is induced by the three-body interaction during the first merger. In type $\mathrm{D}$, significant gas accretion occurs before the first merger. Here, the discrimination between types $\mathrm{B}$ and $\mathrm{C}$ is determined by subsequent mergers. However, in this study, since simulations are terminated at the first merger, types $\mathrm{B}$ and $\mathrm{C}$ are not discriminated. From Figure 2, the merger mechanisms in the GW150914 event is likely to be type B or C, that is, the interplay of gas drag-driven and three-body driven merger or purely three body-driven merger.

Next, we discuss preferable environments for our scenario. Because the environments could vary within $10^{8} \mathrm{yr}$ due to the active galactic nucleus (AGN) duty cycle and/or the galactic rotation timescale, the merger time should be shorter than $10^{8} \mathrm{yr}$. Besides, the merger time is almost inversely proportional to the gas density (Tagawa et al. 2015). From these conditions, the gas number density should be higher than $10^{5} \mathrm{~cm}^{-3}$. Therefore, galactic nuclear regions and dense interstellar cloud cores are favorable sites for the merger of non-binary isolated BHs.

\section{Event rates}

We roughly estimate the event rates based on our scenario for the first observing run of LIGO advanced detectors. First, we estimate the event rates for the merger in dense interstellar cloud cores. Roughly, there are $10^{8}$ stellar-mass BHs in each Milky Way (MW)-sized galaxy (Remillard \& McClintock 2006). Here, the volume of the MW Galaxy is about $10 \mathrm{kpc} \times 10 \mathrm{kpc} \times 1 \mathrm{kpc}=100 \mathrm{kpc}^{3}$, and the total volume of all giant molecular clouds (GMCs), whose number is supposed to be about 1000 in the MW Galaxy, is about $10 \mathrm{pc} \times 10 \mathrm{pc} \times 10 \mathrm{pc} \times 1000=10^{-3} \mathrm{kpc}^{3}$. Thus, the expected number of BHs that exist 
in GMCs is roughly $10^{8} \times\left(10^{-3} \mathrm{kpc}^{3} / 100 \mathrm{kpc}^{3}\right)=1000$. If we consider the Poisson distributions, about 100 GMCs are likely to possess multiple BHs. Since the merger time in GMCs of $n_{\text {gas }} \sim 10^{6} \mathrm{~cm}^{-3}$ is about $20 \mathrm{Myr}$ (Tagawa et al. 2015), the merger rate in a MW-sized galaxy is $100 / 20 \mathrm{Myr}=5 \mathrm{Myr}^{-1}$. For the binary with total mass of $65 M_{\odot}$, we adopt a horizon distance of $D_{h} \approx 3 \mathrm{Gpc}(z \approx 0.3)$ for the first advanced LIGO observation run (Belczynski et al. 2016), corresponding to a comoving volume $V_{c} \approx 50 \mathrm{Gpc}^{3}$. By assuming the number density of the MW-sized galaxies as about $2 \times 10^{6} \mathrm{Gpc}^{-3}$, the number of MW-sized galaxies involved in this volume is $50 \mathrm{Gpc}^{3} \times 2 \times 10^{6} \mathrm{Gpc}^{-3}=1 \times 10^{8}$. We also assume that ratio of massive BHs to all BHs is 0.1 , and the fraction of massive $\mathrm{BH}$ pairs to all $\mathrm{BH}$ pairs is supposed to be 0.01 . From these assumptions, the event rates in GMCs is estimated to be $5 \mathrm{Myr}^{-1} \times 0.01 \times 1 \times 10^{8}=5 \mathrm{yr}^{-1}$.

Next, we estimate the event rates for the merger in galactic nuclear regions (GNRs). In the Milky Way Galaxy, about $3 \times 10^{6}$ stars exist in 1 pc from the supermassive BH (Alexander 2005). If we assume that about one $\mathrm{BH}$ exists per 1000 stars, $3 \times 10^{3} \mathrm{BHs}$ may exist in GNRs. Based on our simulations, we envisage that all stellar-mass BHs in GNRs can merge with each other in the AGN phase $(\sim 100 \mathrm{Myr})$ because of the high density of GNRs in the AGN phase. From these conditions, we estimate that the event rates in GNRs are $\sim 0.3 \mathrm{yr}^{-1}$ by following the estimate of the event rates in GMCs.

Thus far, we estimate the event rates of mergers for the Pop I/II remnant BHs. Besides, we also estimate that the event rates of mergers for Pop III remnant BHs whose number is assumed to be $\sim 10^{6}$ in MW-sized galaxies are $\sim 3 \mathrm{yr}^{-1}$ in GMCs and $\sim 0.1 \mathrm{yr}^{-1}$ in GNRs. As a result, the event rates are about $\sim 8 \mathrm{yr}^{-1}$ for the mergers in GMCs and $\sim 0.4 \mathrm{yr}^{-1}$ for the mergers in GNRs. Here, since our estimates contain large uncertainties such as the uncertainty of the number and distribution of BHs, the estimated event rates are possibly accompanied by the uncertainties of one or two order of magnitude. Furthermore, our scenario prefers disk galaxies as host galaxies of GW events, because gas rich environments are required. To confirm our scenario, additional GW observations are desired.

\section{Acknowledgements}

This research was supported by a grant from the Hayakawa Satio Fund awarded by the Astronomical Society of Japan. Numerical computations and analyses were carried out on Cray XC30 and computers at Center for Computational Astrophysics, National Astronomical Observatory of Japan, respectively. This research was also supported in part by Interdisciplinary Computational Science Program in Center for Computational Sciences, University of Tsukuba, and Grant-in-Aid for Scientific Research (B) by JSPS (15H03638).

\section{References}

Abbott, B., et al., 2016, Phys. Rev. Lett., 116, 061102

Alexander, T., 2005, Physics Reports, 419, 65

Belczynski, K., Holz, D. E., Bulik, T., \& O'Shaughnessy Richard., 2016, Nature, 534, 512

Remillard, R. A. \& McClintock, J. E., 2006, ARA $\& A$, 44, 49

Tagawa, H., Umemura, M., Gouda, N., Yano, T., \& Yamai, Y., 2015, MNRAS, 451, 2174

Tagawa, H., Umemura, M., \& Gouda, N., 2016, MNRAS, 462, 3812 